\title{
VALORES PROFESIONALES DE ENFERMERÍA EN EL POSMODERNISMO: UNA REVISIÓN SISTEMÁTICA
}

\begin{abstract}
Margarita Poblete Troncoso ${ }^{1}$, Marcelo Correa Schnake², Pamela Aguilera Rojas ${ }^{1}$, Juan Carlos González Acuña ${ }^{3}$

Resumen: La sociedad postmoderna basada en un modelo neoliberal cuestiona el fuerte componente valórico característico del cuidado profesional de enfermería. Ante esta situación surge la interrogante: ¿Cómo se enfrenta la formación valórica en los estudiantes de enfermería y cómo se viven estos en la práctica del cuidado? Objetivo: Analizar qué se ha investigado en los últimos diez años sobre la formación ética en estudiantes de enfermería y en la práctica valórica de estos profesionales en una sociedad postmoderna. Metodología: Revisión sistemática mixta de carácter narrativo que considero los últimos diez ańos (2008-2018), incluye 76 artículos de los cuales se seleccionaron 48 para su análisis. Se revisaron las bases de datos: ProQuest, ProQuest education Database, ProQuest Religión Database, Ebsco, Cuiden, Dialnet plus, Latindex, Scielo, SCOPUS y Google académico. Conclusiones: El postmodernismo trastoca los valores profesionales, tanto en la práctica del cuidado como en la formación de los futuros profesionales, por ello se requiere fortalecer y adecuar los valores tradicionales entre la academia y la clínica, centrando la formación en el profesor como modelo de la práctica profesional.
\end{abstract}

Palabras clave: educación en enfermería, ética en enfermería, posmodernismo

\section{Professional values of nursing in the postmodernism: a systematic revision}

\begin{abstract}
Abtract: The postmodern society based on a neoliberal model questions the strong value component characteristic of professional nursing care. Faced with this situation, the question arises: How is valuation training faced by nursing students and how are these students living in the practice of care? Objective: To analyze what has been researched in the last ten years about the ethical formation in nursing students and the value practice of these professionals in a postmodern society. Methodology: Mixed systematic review of a narrative nature that I consider the last ten years (2008-2018), includes 76 articles, of which 48 were selected for analysis. The databases were revised: ProQuest, ProQuest education Database, ProQuest Religion Database, Ebsco, Cuiden, Dialnet plus, Latindex, Scielo, SCOPUS and Google academic. Conclusions: Postmodernism upsets professional values, both in the practice of care and in the training of future professionals, for this reason it is necessary to strengthen and adapt the traditional values between the academy and the clinic, focusing the training on the teacher as a model of professional practice
\end{abstract}

Keywords: nursing education; nursing ethics; postmodernism

\section{Valores profissionais de enfermagem no posmodernismo: uma revisão sistemática}

Resumo: A sociedade pósmoderna baseada em um modelo neoliberal questiona o forte componente de valor característico do cuidado profissional de enfermagem. Diante desta situaçáo surge a pregunta: Como se enfrente a formação de valores nos estudantes de enfermagem e como isto é vivido na prática do cuidado? Objetivo: Analisar o que se investigou nos últimos dez anos sobre a formação ética nos estudantes de enfermagem e na prática de valor destes profissionais em uma sociedade pósmoderna. Metodologia: revisão sistemática mista de caráter narrativo que considere os últimos dez anos (2008-2018), incluindo 76 artigos dos quais se selecionaram 48 para análise. Foram revisadas as bases de dados: ProQuest, ProQuest education Database, ProQuest Religión Database, Ebsco, Cuiden, Dialnet plus, Latindex, Scielo, SCOPUS e Google acadêmico. Conclusóes: O postmodernismo transtorna os valores profissionais, tanto na prática do cuidado como na formação dos futuros profissionais, por isso se requer fortalecer e adequar os valores tradicionais entre a academia e a clínica, centrando a formação no professor como modelo da prática profissional.

Palavras chave: educação em enfermagem; ética em enfermagem; postmodernismo

\footnotetext{
${ }^{1}$ Facultad de Ciencias de la Salud, Universidad Católica del Maule, Chile Correspondencia: mpoblete@ucm.cl

${ }^{2}$ Facultad de Ciencias Religiosas y Filosóficas, Universidad Católica del Maule, Chile

${ }^{3}$ Escuela de Enfermería Universidad Autónoma de Chile, sede Talca, Chile
} 


\section{Introducción}

Desde la tradición disciplinar, la formación profesional en enfermería se ha caracterizado por sus raíces valóricas, lo que se evidencia en los programas de formación, en los estudios realizados a esta profesión y en la misma práctica cotidiana de la enfermería $(1,2)$. Gracias a ello, la sociedad identifica la profesión de enfermería con una actitud altruista, solidaria con los que sufren y de gran entrega personal en su labor de cuidar. Atributos entendidos también como propios por los mismos profesionales de la enfermería(3).

Sin embargo, en esta última década, se percibe en las nuevas generaciones de profesionales de la salud una comprensión valórica distinta, en la que prima el bienestar propio como condición mínima exigida para realizar sus trabajos o deberes profesionales(4). También, se reconoce en ellas una fuerte influencia del individualismo y pragmatismo, lo que va asociado a la obtención de resultados personales inmediatos y a un bajo interés por profundizar los fundamentos disciplinares(5-7). Esta nueva actitud es considerada como contraria a la valoración en la formación y en el ejercicio profesional tradicional de la enfermería.

Algunos autores atribuyen este cambio de actitud al relativismo moral imperante en las actuales generaciones, lo que sería consecuencia de un cambio social mayor. En este sentido, los aspectos éticos de la profesión se mecanizan con la aplicación de técnicas de análisis y decisiones ante problemas o dilemas éticos en salud(8), sin profundizar mayormente en los fundamentos teóricos de los mismos (teorías, principios o valores) $\mathrm{y}$ en las consecuencias que se derivan de ellos(9). En síntesis, se observa una aplicación técnica de la ética, basada en protocolos a seguir más que en la responsabilidad subjetiva en la toma de decisiones profesionales. Esta actitud es considerada por algunos autores como consecuencia de un modelo socioeconómico de mercado neoliberal, que prioriza el individualismo y las relaciones pragmáticas y utilitarias por sobre las relaciones comunitarias y de sentido social(10).

Partiendo de la situación esbozada, la presente investigación se planteó conocer qué se ha investigado estos últimos diez años sobre la for- mación ética de los estudiantes de enfermería y de la práctica valórica de estos profesionales en ejercicio, teniendo como contexto el cambio cultural que enfrenta el occidente iberoamericano. Para ello, se realizó una revisión bibliográfica en artículos científicos, con la finalidad de identificar los problemas que enfrenta la disciplina de enfermería en la formación y aplicación de valores en la práctica del cuidado.

\section{Metodología}

Se llevó a cabo una revisión sistemática mixta de carácter narrativo, que incluye 90 artículos publicados entre 2008 y 2018, de los cuales se seleccionaron 68 artículos para su análisis. Esta revisión se realizó en las siguientes bases de datos: ProQuest, ProQuest education Database, ProQuest Religión Database, Ebsco, Cuiden, Dialnet plus, Latindex, Scielo, SCOPUS y Google académico. En la búsqueda se utilizaron los siguientes descriptores y sus combinaciones: (Cambios OR changes) AND (values OR valores) AND ("nursing education" OR "formación en enfermeria"); (values OR valores) AND ("nursing education" OR "formación en enfermeria"); (values AND "nursing education") (values AND "nursing education").

Se seleccionaron los artículos que estaban indexadas en Scielo, WOS y SCOPUS, por ser las bases de mayor impacto académico. Para la revisión de los artículos seleccionados se elaboró una matriz que incorporaba criterios de selección, indexación, caracterización y análisis del artículo. En una primera revisión se clasificó el tipo de publicación de acuerdo con la metodología utilizada por los autores: investigaciones cualitativas o cuantitativas, u otro tipo de manuscrito.

Posteriormente se organizó el material en tres temáticas:

1. Contexto social, económico y político que afecta los valores en el sistema de salud, de los beneficiarios y de los profesionales.

2. Ejercicio profesional en enfermería y su relación con los valores.

3. Formación en valores en enfermería: focalizado 
Tabla Nº 1 : Selección de artículos por metodología y temática

\begin{tabular}{|c|c|c|c|c|}
\hline Temas & $\begin{array}{c}\text { Investigación } \\
\text { cuantitativa }\end{array}$ & $\begin{array}{c}\text { Investigación } \\
\text { cualitativa }\end{array}$ & $\begin{array}{c}\text { Reflexión, } \\
\text { ensayo u otro }\end{array}$ & Total \\
\hline $\begin{array}{c}\text { 1. El posmodernismo } \\
\text { como contexto del } \\
\text { cambio valórico en } \\
\text { los profesionales de la } \\
\text { salud. }\end{array}$ & 4 & 4 & 7 & 15 \\
\hline $\begin{array}{c}\text { 2. Ejercicio profesional } \\
\text { en enfermería y su } \\
\text { relación con los } \\
\text { valores. }\end{array}$ & 5 & 4 & 14 & 23 \\
\hline $\begin{array}{c}3 . \text { Formación en } \\
\text { valores en enfermería: } \\
\text { focalizada en estrategias } \\
\text { metodológicas y } \\
\text { valores en estudiantes y } \\
\text { académicos. }\end{array}$ & 5 & 15 & 18 & 38 \\
\hline Total & 14 & 23 & 39 & 76 \\
\hline
\end{tabular}

en estrategias metodológicas y valores en estudiantes y académicos.

\section{Resultados}

En la tabla No 1 se presenta un resumen de los artículos.

Siguiendo los criterios seleccionados, esta matriz entrega los contenidos que a continuación se presentan sobre la base de las temáticas señaladas.

\section{El posmodernismo como contexto del cam- bio valórico en los profesionales de la salud}

La literatura revisada nos presenta un panorama de la sociedad contemporánea que permite contextualizar la realidad de los profesionales de la salud y, en particular, de la vivencia y formación valórica de los mismos en las instituciones de educación superior.

Al profundizar en el actuar, el rol social y el propósito del profesional de enfermería, se reconoce un conflicto en la identidad de la profesión. Tomando en cuenta lo declarado, tanto en las bases epistemológicas y en los códigos de ética como en las diversas propuestas curriculares de las casas formativas, se reconocen ciertos valores altruistas en esta profesión. De modo que la entrega al otro por compasión, el sacrificio personal y la responsabilidad profesional han sido los valores identificados en la construcción social de esta disciplina $(1,9,8)$.

La vivencia de estos valores se da en un contexto social, político y económico que está en proceso de cambio(11). En la literatura se identifica el origen de estos valores en una sociedad marcada por la centralidad de la comunidad, de inspiración cristiana, que se reinterpreta desde la racionalidad moderna, secularizando estos valores y manteniéndolos como fundamento de la profesión.

No obstante, en la literatura más reciente, se evidencia un conflicto en la sociedad posmoderna, puesto que se cuestiona el sentido de la vocación de los profesionales de la salud y su actual práctica clínica a partir de un nuevo ideal profesional. Este está marcado principalmente por el éxito y la excelencia individual por sobre el servicio al desarrollo comunitario. Estos nuevos valores se miden y evidencian a través de los logros económicos y la especialización técnica, desvinculándolos de los aspectos humanizadores y de una visión integral del ser humano $(12,9,8)$.

Victoria Camp grafica esta realidad del siguiente modo: "El ethos profesional reducido a un ethos 
mercantilista y burocrático no atiende al cultivo de los valores o virtudes que debería exhibir la práctica sanitaria" (12:5).

Desde un punto de vista explicativo, autores como Rosales-Nieto(13) y Duque(14) hablan de una modificación de los valores sociales producto de dos visiones de sociedad contrapuestas entre sí. Este binomio social presenta, por un lado, una sociedad tradicional, caracterizada por una comprensión religiosa o racionalista, que sostiene una visión autoritaria y tradicional, vinculada a valores como la obediencia y la sumisión; y una sociedad posmoderna, que sostiene una visión más bien individualista, que promueve los valores como la felicidad personal, la autorrealización y la participación. Otros autores, parafraseando a Ortega y Gasset, califican a este fenómeno como "desorientación vital", haciendo referencia a que las aspiraciones y creencias de los actuales profesionales de la salud son concebidas con marcada individualidad en función del entorno(15).

En definitiva, los autores reconocen la existencia de un cambio de valores producto de un cambio sociocultural que ha transformado el sistema occidental de valores de origen judeocristiano(13), lugar donde tuvieron una significativa influencia en los valores de los profesionales de la salud, pasando en la posmodernidad a establecer relaciones sociales y económicas que edifican primero al sujeto autónomo y exitoso, basado principalmente en una formación técnico-científica y débilmente formado en el humanismo. En respuesta a este movimiento, algunos autores proponen volver a los orígenes de las profesiones de salud para retomar los valores propios que las deben susten$\operatorname{tar}(16-20)$.

Este cambio de contexto sociocultural se evidencia en la disociación entre la capacitación científico-técnica y los nuevos valores profesionales en los servicios de salud(12). La relevancia de esta situación ha llegado a tal extremo que la bioética ha debido encargarse de este complejo escenario, con la finalidad de mantener vigentes en la práctica clínica los valores alcanzado por la humanidad(21), valores reconocidos como centrales durante siglos en los profesionales de la salud.

Según unos estudios, tal fenómeno podría ser dimensionado al analizar los alcances de la posmodernidad sobre el individuo y su nueva comprensión de la realidad. Es así como, en esta nueva época, podemos hablar de un ser humano con diferentes necesidades, articuladas para alcanzar su propia satisfacción. Desde esta mirada, y como partícipes de la sociedad, los profesionales se han adaptado a las nuevas exigencias, llevándolos a modificar sus propios valores para dar respuesta a una nueva sociedad, olvidando el diálogo con el pasado y la tradición para hacer frente a las demandas vigentes. Lo que se traduce en una pérdida de identidad profesional(10).

La lógica posmoderna presenta múltiples paradojas en las prestaciones. Una de ellas se genera entre la igualdad social y la libertad individual para el acceso en salud. En este sentido, a pesar de la gran necesidad de justicia distributiva, la gratuidad no es vista con buenos ojos, ya que los individuos desean elegir sus prestaciones(13). La sociedad contemporánea reconoce y privilegia otras singularidades de la relación clínica, como son el tiempo y los recursos, los modelos de atención y la relación profesional-paciente, para las cuales se crean respuestas innovadoras y se desarrollan nuevas capacidades para enfrentarlas, facilitando con esto la elección del paciente, como por ejemplo en la relación prestador-cliente, lo que también trae consigo nuevos derechos del consumidor y nuevas obligaciones en la responsabilidad profe$\operatorname{sional}(10)$.

De igual modo, en la posmodernidad se reconoce la importancia de la salud pública, pero bajo la fuerte influencia del desarrollo económico de la producción farmacéutica (producción, precio, calidad). En este sentido, la industria lleva las pautas de lo que es necesario para la población, creando necesidades y dando a la vez respuestas a las mismas, siempre teniendo presente los nuevos valores basados en la imagen, el consumo y el hedonismo(14).

De este modo, la influencia de la posmodernidad exige a la sociedad contemporánea un cambio de paradigma en la comprensión y en el sistema de salud. El que se verifica primero en el paso de un modelo médico-científicista a un modelo biopsico-social, para luego derivar en un modelo bioeticista enmarcado en la legalidad de la ética 
sanitaria y del derecho del paciente, ambos configurados y regidos por los intereses económicos liberales(10).

En consecuencia, y contrariamente a lo que se podría esperar, para los distintos pensadores, la misma posmodernidad, al no hacer el esfuerzo por alcanzar, proponer ni defender valores morales superiores, ha legitimado la necesidad de una idea de autosacrificio, generando la necesidad de defender valores altruistas(10).

\section{Segundo criterio: valores y ejercicio profesion- al de enfermería}

Los estudios y las reflexiones en estos últimos diez años abordan la relación entre los valores y el ejercicio profesional en enfermería en los siguientes temas: el autoconocimiento de los propios valores por parte del profesional, la importancia de las relaciones interpersonales y de la comunicación en la práctica del cuidado, la priorización de los valores colectivos por sobre los individuales y la necesidad de formación ética permanente del profesional de enfermería. Estos temas han sido investigados principalmente en los profesionales que trabajan en unidades críticas, por lo complejo y sensible de su actividad, y, de manera global, se ha indagado sobre el impacto de la cultura organizacional de los hospitales públicos en la vivencia de los valores éticos por parte de los profesionales de la salud, y en particular de los de enfermería.

Los autores destacan, en primer lugar, la importancia del autoconocimiento de los propios valores por parte del profesional de la salud al momento de cuidar a las personas. A esta capacidad personal se atribuye una relevancia fundamental, reconociendo que, sin ella, no se podría hablar de una actuación ética profesional responsable. Por ejemplo, se señala que esta capacidad se exige especialmente cuando los cuidados se realizan con personas que padecen una enfermedad estigmatizada, de tal forma de asegurar el respeto de los valores del "otro". Sin embargo, al margen de la capacidad de autoconocimiento, se reconoce en general en los profesionales de enfermería una marcada vocación de servicio y compromiso en el cuidado de la persona, cuestión que se evidencia incluso sin que estos tengan clara conciencia de ello(22,23).
Dentro de los profesionales de la salud, el profesional de enfermería que trabaja en unidades críticas es el más estudiado en los aspectos valóricos, dadas las características propias de su trabajo. El profesional, aquí, enfrenta con mayor intensidad conflictos morales en relación al sufrimiento de los enfermos, manifestando una conducta disociativa entre sus funciones técnicas y las relaciones personales, tanto en el trato con el usuario como con su familia(24).

La importancia de las relaciones interpersonales y la trascendencia de la comunicación en la práctica del cuidado en estas unidades es constantemente destacada. Los autores señalan que, si bien la tecnología ha significado un progreso para la salud de las personas, esta se ha convertido en una barrera para las relaciones interpersonales. Un ejemplo de ello es la preferencia que manifiestan los profesionales por atender pacientes sedados, evitándose así el encuentro personal con el sufrimiento del otro $(25,26)$.

Otro aspecto estudiado, que afecta los valores en el ejercicio profesional, es el tema de la cultura organizacional en los hospitales públicos. La cultura institucional es percibida por los profesionales de enfermería como una jerarquía rígida, con centralización del poder. Estableciendo relaciones competitivas que dificultan el trabajo en equipo, generando escasa satisfacción laboral y entorpeciendo la promoción de las relaciones interpersonales. En razón de esta cultura organizacional, las instituciones de salud tienen un modelo curativo que termina siendo un obstáculo para el desarrollo del ser de la enfermería y genera conflictos valóricos difíciles de conciliar(27).

Como resultado del despliegue del escenario socioeconómico liberal, se evidencia una contraposición entre las actuales tendencias de la cultura organizacional con los valores laborales de la profesión de enfermería, que prioriza los valores colectivos versus los individuales. En tal sentido, en enfermería prevalecen los valores que están al servicio de un cuidado humanizado, tales como el respeto, el perdón, la responsabilidad, la humildad y la generosidad para brindar cuidados dignos a los usuarios(28). Otros estudios destacan como valores comunes a la práctica de enfermería la verdad, el amor, la responsabilidad, la vocación, 
el respeto, la amistad, el conocimiento y la puntualidad(11).

$\mathrm{Al}$ respecto, en relación a los procesos éticos y denuncias recibidas en enfermería, se señala que las iatrogenias, el ejercicio ilegal de profesiones, las relaciones interprofesionales conflictivas y la responsabilidad profesional del enfermero son las denuncias más frecuentes. Por tanto, es necesario reflexionar e intervenir en la práctica y en la formación continua de los profesionales en los espacios institucionales de la salud(29), promoviendo la apropiación de las propias ideas, creencias y valores en el ejercicio profesional, con la finalidad de fortalecer las convicciones personales y acentuar la responsabilidad profesional.

\section{Tercer criterio: formación en valores en los es- tudiantes de enfermería}

Los estudios de esta última década informan que el nuevo contexto socioeconómico ha impactado, tanto en el quehacer de la enfermería, como en la formación de los futuros profesionales. Respecto de este último asunto, la bibliografía presenta diversos tópicos. Por un lado, se destaca la pérdida de valores tradicionales por parte de los estudiantes y se reconoce cambios valóricos intergeneracionales que dificultan el aprendizaje y, por otro, la deficiente formación académica y escasa preparación metodológica de los profesores en la educación superior. En respuesta a esta problemática se proponen diversas estrategias metodológicas, priorizando, de entre ellas, el aprendizaje de servicio en la comunidad. Desde esta metodología y con una actitud tolerante, se pretende asumir positivamente el cambio valórico de las nuevas generaciones.

En estos últimos años, en Latinoamérica, se han invisibilizado los valores tradicionales en las profesiones de la salud. Esto se entiende como resultado de la influencia del modelo neoliberal en la comprensión del hombre moderno y de su rol profesional, como se ha señalado. Es así que se comprende al sujeto posmoderno como aquel que prioriza su autonomía por sobre cualquier otro principio, lo que le lleva a relativizar los valores tradicionales. La consecuencia de esta nueva actitud en las profesiones de salud se evidencia en cuestionar lo establecido y, a la vez, en menospre- ciar la sabiduría de aquellos que tienen la experiencia en la práctica del cuidado $(8,14,15)$.

Esta dificultad se evidencia en los estudiantes que viven inmersos en esta relatividad valórica, situación que les complejiza asumir valores tradicionales que caracterizan a la profesión de enfermería, como, por ejemplo, el amor al trabajo, la responsabilidad y la abnegación por el cuidado de la persona(30). A pesar de lo señalado, es frecuente encontrar que estos valores se manifiestan en los estudiantes de enfermería(31), lo que se atribuye al lugar primordial que se da a la formación ética en el curriculum de enfermería(32).

En el proceso formativo del profesional de enfermería, los autores identifican al profesor como la figura central y de mayor responsabilidad. El rol del académico es entendido como modelo en la práctica del cuidado y, a su vez, experto pedagogo y estratega para formar en valores. El problema radica en que los académicos presentan importantes deficiencias en la formación en valores y cuentan con escasas estrategias formativa para un adecuado ejercicio pedagógico(33-35).

Los estudios también reconocen que los valores sociales que predominan en esta última década afectan a todos los actores de la educación superior, y que no se puede hacer recaer sobre un solo agente la responsabilidad de la formación profesional. En razón de ello, se postula la educación como un trabajo en conjunto entre varios actores: las instituciones prestadoras de servicios de salud, las instituciones de educación superior, los académicos y los estudiantes. Y se considera que el lugar más adecuado para llevar a cabo esta formación es en la práctica clínica(35).

Ello se basaría en la convicción de que la formación en valores no puede enseñarse solo en la teoría, se requiere de una experiencia real. La práctica clínica permite al estudiante el descubrimiento de los valores que conlleva la experiencia profunda del cuidar al otro. En este contexto, la figura del profesor pasa a ser el modelo por excelencia, puesto que forma y acompaña al estudiante en el aprendizaje de ser enfermero(36-39).

Complementariamente a la práctica clínica, se hacen propuestas metodológicas, como el nom- 
brado "aprendizaje servicio", las que acentúan una formación integral en los valores de la enfermería. Esta metodología permite a los estudiantes realizar actividades de servicio a la comunidad visibilizando las necesidades reales de la sociedad, lo que les posibilita utilizar diversas herramientas académicas en función de desarrollar un aprendizaje de los siguientes valores sociales y que, a su vez, son fundamentales en la profesión: la justicia, la responsabilidad, la solidaridad y la vocación de servicio $(40,41)$.

Otra propuesta, en el contexto de las prácticas clínicas, son las metodologías activas, que permiten relacionar, de modo integral y armónico, la formación valórica de los estudiantes con el conocimiento y el raciocinio clínico propio de la profesión. Estas metodologías exigen utilizar espacios motivadores y una comunicación empática en el intercambio de saberes entre profesores y estudiantes, destacándose el diálogo como una de estas estrategias(42).

En estos últimos años, frente a una creciente diversidad y relatividad moral de la sociedad, se ha priorizado una formación en el diálogo, con la finalidad de llegar a un consenso que permita actualizar la comprensión y la vivencia de los valores tradicionales de la enfermería(43).

Esta postura es corroborada por evaluaciones realizadas a los estudiantes. En ellas, los investigadores demuestran, en diversos estudios, que no corresponde afirmar que los estudiantes no poseen valores sociales. Por el contrario, las mediciones tomadas con diferentes escalas muestran que los jóvenes se identifican con valores sociales, tales como la dignidad humana, la igualdad, la justicia, el altruismo, la estética y, en relación a los valores personales, aprecian dar sentido a la vida, a la libertad, al auto respeto, a la seguridad familiar y al trabajo $(16,44,15)$. De igual modo, los resultados de estas investigaciones confirman la necesidad de continuar evaluando la formación valórica en las futuras generaciones, con la finalidad de actualizar los currículos y, de ser necesario, modificar las estrategias que apoyen esta formación activa y dialógica.

Actualmente, la educación superior está transitando a un modelo por competencias y las ma- llas curriculares se están innovando y rediseñando constantemente. En este contexto, se debe tener en consideración lo sugerido por algunos autores(45): incorporar la educación moral y la formación en valores como un eje curricular. Sin embargo, hoy la formación en ética es compleja y hay diversidad de opiniones sobre los valores a formar en los futuros profesionales(46).

Por ello, para orientar esta formación se requiere que los valores incorporados en el proyecto curricular sean asumidos tanto por las instituciones prestadoras de servicio como por las universidades, buscando relacionar coherentemente la educación profesional con la formación ciudadana de sujetos críticos del contexto social, pero, a su vez, reflexivos y respetuosos con el otro $(47,48,16-18)$.

\section{Conclusiones}

De la presente indagación bibliográfica, se puede concluir que se evidencia un significativo número de artículos publicados en revistas indexadas en los últimos 10 años; sin embargo, se reconocen en ellos solo unos cuantos autores que abordan de modo más sistemático y constante la problemática. Lo común es encontrar publicaciones aisladas al respecto, sin que los autores se citen o asocien en ello. Por consiguiente, para abordar con mayor responsabilidad y eficacia esta temática habría que conformar equipos interdisciplinares e internacionales de investigadores que perduren en el tiempo.

Por otro lado, se reconoce que el posmodernismo trae consigo una comprensión de la realidad que afecta el ejercicio y la formación profesional en salud, donde se cuestionan los valores tradicionales de la profesión, siendo una de las principales dificultades el antagonismo que se suscita entre los valores profesionales tradicionales, presentes en sus códigos de ética, y los valores socioeconómicos que asumen los servicios de salud, conducidos por un modelo económico neoliberal. Lo que se ha traducido en la negación de los valores morales más básicos, para dar paso aquellos que inculta el sistema económico, teniendo como resultados la maximización del uso de los recursos y la disminución de la calidad en el cuidado. Ante ello, se ve necesario realizar un análisis crítico que permita comprender, en mayor profundidad, las 
causas, los efectos y el impacto de este este nuevo contexto posmoderno en salud para asumir creativamente las nuevas exigencias éticas a las profesiones de la salud.

En relación con la práctica de enfermería, se evidencian problemas en tres niveles: deficiencias en el cuidado de las personas, deterioro en las relaciones interpersonales entre el personal y confusión en la formación de las nuevas generaciones. Respecto de ello, se requiere proyectar una investigación interdisciplinar vinculada con el autocuidado del personal de salud, de modo de conocer y crear nuevas estrategias que atiendan la compleja situación del quehacer profesional actual.

Acerca de la formación en valores en los estudiantes de enfermería, los estudios muestran que estos poseen valores sociales y personales jerarquizados desde su autonomía y conveniencia personal, y proponen como modelo de formación en la práctica del cuidado del otro a un profesor que posea características personales y pedagógicas que den cuenta de una actitud crítica, reflexiva, dialogante y tolerante. Así, también, los estudios presentan como mejores estrategias las "metodologías de aprendizaje servicio", "metodologías activas" y la "educación en el diálogo". En síntesis, la educación de los nuevos profesionales de la salud requiere de un trabajo entre todos los actores sociales representados en los sistemas de salud, las clínicas y las universidades. Este punto se evidencia como un escenario complejo, puesto que, si bien cada uno de ellos contribuye al mismo objetivo común: la salud de la población, cada uno también lo aborda desde una perspectiva diferente, desconociendo las otras. $\mathrm{Al}$ respecto, se reconoce en esta investigación la necesidad de estudiar las relaciones estructurales entre la gestión, la formación y el ejercicio profesional de las carreras de salud, para hacer una propuesta formativa en ética que articule dichas dimensiones en este nuevo escenario posmoderno. 


\section{Referencias}

1. Quiala M. Enfermería, formación en valores. Bioética 2013; 13(1): 20-25.

2. Vargas Z. (2013). Valores de enfermería en la práctica profesional. Revista Médica Electrónica Portalesmédicos.com. Acceso el 16 de abril 2017. En: www.revista-portalesmedicos.com/revista-medica/valores-enfermería-practica-profesional/

3. Campos E, Cabral M. Valores de la enfermería como práctica social. Latino-Am. Enfermagem 2013; 21(3): 1-9.

4. Jiménez-López FR, Roales-Nieto JG, Vallejo-Seco G, García-Vargas GR, Lorente-Molina MT, Granados-Gámez G. Valores personales en estudiantes y profesionales de Enfermería. Aquichan 1 de mayo de 2015; 15(1): 105-15.

5. Programa de las Naciones Unidad para el Desarrollo, (PNUD). Desarrollo humano en Chile. Los tiempos de la politización. 2015. Disponible en: http://desarrollohumano.cl/idh/informes/2015-los-tiempos-de-la-politizacion/

6. León Correa FJ. Enseñar bioética: Cómo trasmitir conocimientos, actitudes y valores. Acta Bioethica 2008; 14(1).

7. León Correa FJ. Antecedentes y situación de la enseñanza de la bioética en Chile. En: Vidal SM, (editora). La educación en bioética en América Latina y el Caribe: experiencias realizadas y desafíos futuros. UNESCO; 2012: 219-237.

8. Blasco-León M, Ortiz-Luis SR. Ética y valores en enfermería. Rev. Enferm Inst Mex Seguro Soc. 2016; 24(4): 145-149.

9. Rubio-Domínguez LS. Principios y valores que guían el ejercicio del personal de enfermería. CONAMED 2008; 13(1): 13-15.

10. Daros W, Guida JC, Antiba A. Sociedad posmoderna y salud. Red: Política científica desde Latinoamérica. Simposio Política y Sociedad. Temas biomédicos y Salud, nov 2014.

11. Vera M, Cornejo R, Rivas L, Calizaya J, Zamora O, García J. Significado de los valores profesionales en docentes y estudiantes de enfermería. UNMSM - 2014. Anales de la Facultad de Medicina 12 de octubre de 2016; 77(3): 225.

12. Camps V. Los valores éticos de la profesión sanitaria. Educación Médica enero de 2015; 16(1): 3-8.

13. Roales-Nieto J. Cambio social y cambio personal. Estudio preliminar del cambio de valores en una muestra intergeneracional. International Journal of Psychology and Psychology Therapy 2009; 9(3): 395-420.

14. Duque E. Valores de la sociedad moderna: una visión del cambio social. HOLOS 2017; 32(2): 317-336.

15. Rivas RE, Rivas LA, Bustos ML. Valores expresados en estudiantes de enfermería: Temuco - Chile, 2009. Ciencia y Enfermería 2011; 17(2): 65-75.

16. Díaz M, Castro D, Cuevas B. Valores profesionales en enfermería: Una mirada hacia la formación. En: Rivas RE, Rivas LA, Bustos ML. La educación superior. Humanidades Médicas 2012; 12(2): 289-299.

17. Nunes ECDA, Silva LWS, Pires EPOR. La enseñanza superior de enfermería: implicaciones de la formación profesional para el cuidado transpersonal. Rev. Latino-Am. Enfermagem 2011; 19(2): 252-260.

18. Olivé Ferrer MC. Cuidado humanístico y transpersonal: esencia de la enfermería del siglo XXI. Fundamentos disciplinares. SALUS 2015; 19(3): 20-26.

19. Olivé Ferrer MC, Isla Pera MP. El Modelo Watson para un cambio de paradigma en los cuidados enfermeros. Rev ROL enferm 2015; 38(2): 123-128.

20. Jiménez-López FR, Roales-Nieto JG, Vallejo-Seco G, García-Vargas GR, Lorente-Molina MT, Granados-Gámez G. Valores personales en estudiantes y profesionales de Enfermería. Aquichan 1 de mayo de 2015; 15(1): 105-115.

21. Santana K, Rey Y. Necesidad de la formación de valores en los profesionales de la salud. MENDISAL 2015; 19(3): 297-299.

22. De Carvalho-Dantas F, De Carvalho-Dantas C. Valores intervenientes no cuidado do enfermeiro ao cliente com HIV/ Aids. Aquichan 2014; 14(1): 32-40.

23. Burgos CB. El cuidado en enfermería desde las perspectivas de la ética del cuidado y del género. Investigación y Educación en Enfermería 2013; 31(2): 243-251.

24. Da Silva RC, Ferreira MdeA, Apostolidis T, Sauthier M. Nursing care practices in intensive care: An analysis according to ethics of responsibility. Escola Anna Nery - Revista de Enfermagem 2016; 20(4).

25. Wood-Molina T, Rivas-Riveros E. Conflictividad ética en enfermeras/os de unidades de cuidados críticos en un hospital del sur de Chile. Enfermería Universitaria octubre de 2017; 14(4): 224-234.

26. Ramírez P, Müggenburg C. Relaciones personales entre la enfermera y el paciente. Enfermería Universitaria julio de 2015; 12(3): 134-143.

27. De Carvalho MC, Rocha FLR, Marziale MHP, Gabriel CS, Bernardes A. Work values and practices which characterize the organizational culture of a public hospital. Texto Contexto-Enferm. 2013; 22(3): 746-753.

28. Badillo-Reyes L, López-Castellanos L, Ortiz-Ramírez M. Valores laborales prioritarios en el personal de enfermería. Rev Enferm Inst Mex Seguro Soc. 2012; 20(2): 71-78.

29. Schneider DG, Ramos FRS. Nursing ethical processes in the State of Santa Catarina: characterization of factual ele- 
Valores profesionales de enfermería en el posmodernismo - M. Poblete, M. Correa, P. Aguilera, J. C. González

ments. Revista Latino-Americana de Enfermagem agosto de 2012; 20(4): 744-752.

30. Gómez PRM, Ramos RD, Sánchez SJF, Rodríguez PL. La pérdida de valores en estudiantes de enfermería identificados por sus profesores. Revista de Ciencias Médicas de la Habana 2015; 21(3): 619-627.

31. Véliz A, Dörner A, Ripoll M. Perfil de valores de estudiantes de carreras de salud del sur de Chile. Horizonte Médico (Lima) 2017; 17(2): 48-54.

32. Luengo-Martínez CE, Sanhueza-Alvarado O. Formación del licenciado en Enfermería en América Latina. Aquichan 1 de junio de 2016; 16(2): 240-255.

33. Milanés Céspedes N, Bujardón Mendoza A, Tamarit Castillo O, Valdés Cervantes VM. Planeación estratégica para el fortalecimiento de la ética profesional de Enfermería en la filial Nuevitas. RevHumMed 2010; 10(3).

34. Palencia E, Piña M, Nuñez C. Los docentes universitarios de enfermería y sus valores. Enfermería Global abril de 2011; $10(22)$.

35. Sosa Martínez LI, Espinosa Arencibia A, Corne Carmenate R, Corne Sosa E, Reyes Corne M, Leal FM. Acciones para reforzar los valores responsabilidad y humanismo en los futuros profesionales de la salud. EDUMECENTRO 2016; 8(1): 96-110.

36. Carneiro AD, Da Costa SFG, Pequeno MJP. Disseminação de valores éticos no ensino do cuidar em enfermagem: estudo fenomenológico. Texto \& Contexto - Enfermagem diciembre de 2009; 18(4): 722-730.

37. Ramos FRS, Schoeller SD, Brehmer LCF, Amaral RFC, Melo TAP. Motivaçóes e experiências do ensino da ética/bioética em enfermagem. Av Enferm. 2010; 28(2): 40-47.

38. Guimarães GdeL, Viana LdeO. O valor social no ensino da enfermagem. Escola Anna Nery septiembre de 2012; 16(3): 508-513.

39. Guimarães GdeL, Viana LdeO, De Matos SS, Carvalho DV, Baroni FC de AL. O valor verdade no ensino da enfermagem: um estudo fenomenológico. Revista Gaúcha de Enfermagem marzo de 2013; 34(1): 133-139.

40. Arratia Figueroa A. Ética, solidaridad y “aprendizaje servicio” en la educación superior. Acta Bioethica 2008; 14(1).

41. Garmendia García F, Sánchez Soler E. La disciplina y el aprendizaje como valores en la actividad docente. Rev Cuba Reumatol 2015; 17(Suppl 1): 157-161.

42. Marques LMNS da R. Active methodologies as strategies to develop education in values in nursing graduation. Escola Anna Nery 18 de junio de 2018; 22(3).

43. Enderle C de F, Da Silveira RS, Dalmolin GdeL, Lunardi VL, Avila LI, Dominguez CC. Teaching strategies: promoting the development of moral competence in undergraduate students. Revista Brasileira de Enfermagem 2018; 71(Suppl4): $1650-1656$.

44. Durán Parra M. Valores en los estudiantes de enfermería de la Universidad de Santander. Avances en Enfermería 3 de octubre de 2014; 32(1): 11-24.

45. Montilva M, García M, Torres A, Zapata E, Puertas M, Franco M, Palencia E. Formación de docentes universitarios para la educación moral y valores. Revista Salud, Arte y Cuidado 2012; 5(10): 77-86.

46. Gerber VK de Q, Zagonel IPS. A ética no ensino superior na área da saúde: uma revisão integrativa. Revista Bioética abril de 2013; 21(1): 168-178.

47. Kloh D, De Lima MM, Reibnitz KS. Ethical and social commitment in the teaching plan of nursing education. Texto \& Contexto - Enfermagem junio de 2014; 23(2): 484-491.

48. Sánchez M, Herrera F, Socarras M. La formación de enfermería centrada la enseñanza en los valores del cuidado de la vida y la salud. Rev. Praxis 2012; 8: 138-146.

Recibido: 7 de mayo de 2019

Aceptado: 2 de agosto de 2019 